\title{
Design, synthesis, molecular docking and cytotoxicity evaluation of some novel 5-arylidene-3-(substituted phenyl)-2-(p-tolylamino) -4-imidazolones
}

\author{
Ahmed I. Khodair ${ }^{1}$, Mostafa M. Elbadawi ${ }^{2 *}$, Mohammed T. Elsaady ${ }^{3}$, Khaled R. A. Abdellatif ${ }^{4,5}$ \\ ${ }^{1}$ Department of Organic Chemistry, Faculty of Science, Kafrelsheikh University, Kafrelsheikh, Egypt. \\ ${ }^{2}$ Department of Pharmaceutical Chemistry, Faculty of Pharmacy, Kafrelsheikh University, Kafrelsheikh, Egypt. \\ ${ }^{3}$ Department of Medicinal Chemistry, Faculty of Pharmacy, Beni-Suef University, Beni-Suef 62514, Egypt. \\ ${ }^{4}$ Department of Pharmaceutical Organic chemistry, Faculty of Pharmacy, Beni-suef University, Beni-suef 62514, Egypt \\ ${ }^{5}$ Pharmaceutical Sciences Department, Ibn Sina National College for Medical Studies, Jeddah 21418, Kingdom of Saudi Arabia.
}

\begin{tabular}{l} 
ARTICLE INFO \\
\hline Article history: \\
Received on: $21 / 03 / 2017$ \\
Accepted on: 08/05/2017 \\
Available online: $30 / 09 / 2017$ \\
\hline Key words: \\
4-imidazolones, cytotoxicity, \\
prostate cancer, breast \\
cancer, topoisomerase I.
\end{tabular}

\section{INTRODUCTION}

Worldwide, there are about 14.1 million new cancer cases diagnosed from which 8.2 million people die from cancer. In men, the second most popular cancer type is prostate cancer which represents $15 \%$ of the total cancer cases. The prostate cancer is considered as the fourth leading cause of cancer deaths in men accounting for $6.6 \%$ of the total cancer deaths. Moreover, among women, the most popular site of cancer is the breast accounting for $25.2 \%$ of the total cancer cases also the breast cancer is considered as the first common leading cause of cancer

\footnotetext{
* Corresponding Author

E-mail: Mostafa_elbadawi@pharm.kfs.edu.eg
}

deaths representing $14.7 \%$ of all cancer cases in women (Siegel et al., 2016 and World cancer report). Literature review of 2thioxoimidazolidinone revealed that it is present in many synthetic compounds and it has displayed different pharmacological activities as anticancer, anti-inflammatory and antimicrobial activity (Gauthier et al., 2006; Spicer et al., 2013). 2thioxoimidazolidinone derivatives have been synthesized and evaluated for their cytotoxic activity against many cancer cell lines like prostate and breast cancer cell lines. Also, imidazolones, which are analogues of 2-thioxoimidazolidinones, have been synthesized and found to have different pharmacological activities like anti-inflammatory (Abdellatif and Fadaly, 2017), antimicrobial (Dhingra et al., 2016) and anticancer activity (Kamal et al., 2010; Kumar et al., 2014). 

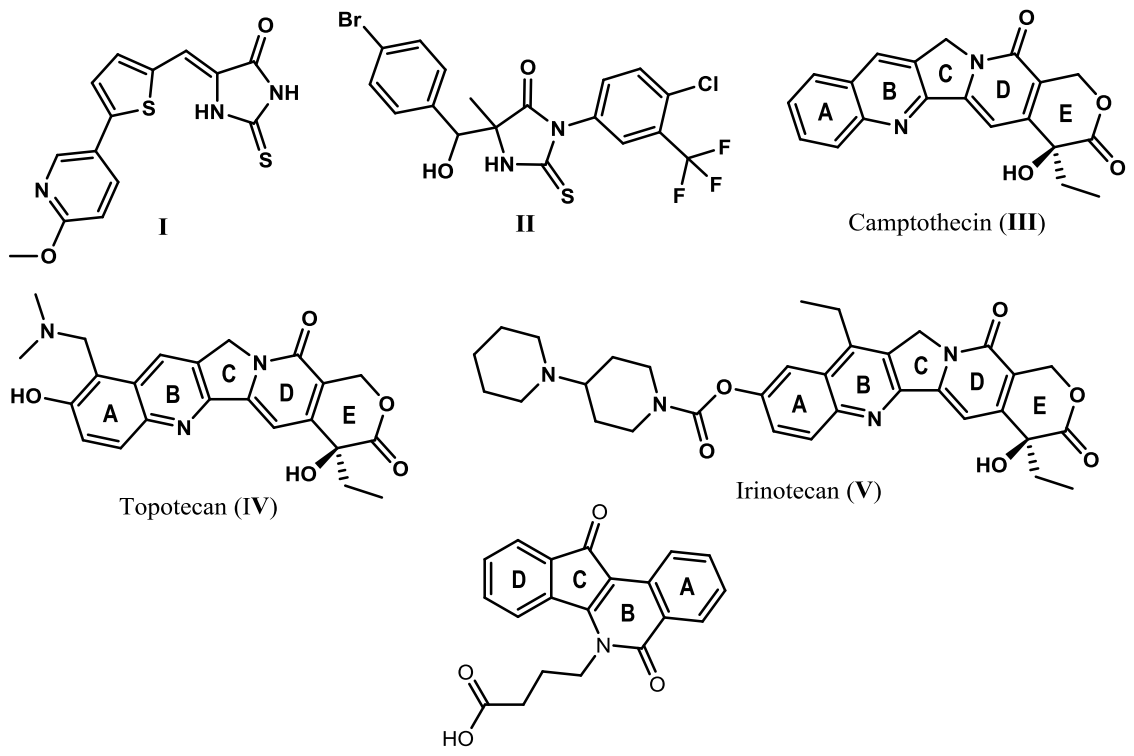

Indenoisoquinoline, MJ238 (VI)

Fig. 1: Human topoisomerase I inhibitors and anti-prostate cancer agent, II.

One of these interesting studies is the design and synthesis of 2-thioxoimidazolidinone derivatives and evaluation of their topoisomerase I inhibitory activity that is considered as a potential chemotherapeutic target. In this study, it was found experimentally and through molecular docking that a derivative of 2-thioxoimidazolidinone (I) had a potent inhibition of human topo I by stabilization of topo I-DNA cleavage complex with $\mathrm{EC}_{50}=$ $10.08 \mu \mathrm{M}$ also it showed a potent cytotoxic activity against MCF-7 (human breast cancer cell line) with $\mathrm{IC}_{50}=11.4 \mu \mathrm{M}$ (Majumdar et al., 2015). Furthermore, other study reported that some aldol derivatives of thioxoimidazolidinone had a potential anti-prostate cancer activity like compound (II) that exhibited good antiproliferative action against androgen insensitive prostate cancer cell line (PC-3) with $\mathrm{IC}_{50}=0.923 \mu \mathrm{M}$ suggesting that this compound may perform its anti-prostate cancer activity through a mechanism other than anti-androgen activity (Khatik et al., 2011). In addition, enzalutamide which is a derivative of 2thioxoimidazolidinone has been approved for the treatment of castration resistant prostate cancer (Jung et al., 2010; Bassetto et al., 2016). Moreover, an interesting study suggested with experimental results that although the sulfur atom in 2thioxoimidazolidinones was removed and replaced by arylamino group forming their analogues, 4-imidazolones, 2-arylamino-4imidazolones exhibited a potent cytotoxic activity against different cell lines as prostate and breast cancer cell lines (Subtel'na et al., 2010).

Topoisomerase I (topo I) is a ubiquitous nuclear enzyme that is involved in solving all topological problems associated with DNA replication and transcription which acts by making a transient break in one strand of DNA followed by rejoining of the DNA strand after passing one strand through each other resulting in relaxation of the supercoiled DNA (Topcu, 2001; Ge et al., 2016; Mazza et al., 2016; Cheng-Kang Peng and Lin, 2017).
Topo I plays a critical role during cell proliferation thus, it has been validated as a major target for the development of anticancer agents as the cancer cells have an accelerated cell proliferation rate which is positively related to the overexpression of topo I in cancer cells than normal ones. In rapidly proliferated cells the replication fork of DNA is thought to collide with the trapped topo I-DNA complex leading to double strand breaks and apoptotic cell death (Topcu, 2001; Staker et al., 2005; Alonso et al., 2016; Beck et al., 2016; Jeon et al., 2016; Park et al., 2017).

Camptothecin (III) and its derivatives, topotecan (IV) and irinotecan $(\mathbf{V})$, are topo I poisons that are currently used clinically for the systemic treatment of colorectal, ovarian, breast and non-small lung cancers. Unfortunately, the clinical use of camptothecin and its analogues are hampered by several drawbacks as rapid inactivation arising from the rapid hydrolysis of lactone ring under the physiological $\mathrm{PH}$, dose-limiting bone marrow suppression and development of resistance resulting from the rapid cellular efflux via the membrane pumps. Non camptothecin topo I inhibitors with better pharmacokinetic properties have been developed such as indolocarbazoles and indenoisoquinolines like MJ238 (VI), now some of them under clinical investigations but not approved yet. Great efforts have been conducted to develop safe and more effective topo I inhibitor with improved stability based on the structure activity relationships (SAR) and binding interactions of the reported topo I inhibitors, (Figure 1)(Hou et al., 2016; Zhang et al., 2016). In addition to camptothecin, indolocarbazoles and indenoisoquinolines, different scaffolds have been reported to have topo I inhibitory activity despite the loss of planar structure that found in the classic topo I poisons like substituted aryl pyridines, indeno-naphthyridines, substituted quinolones and substituted 2thioxoimidazolidinones (Majumdar et al., 2015; Alonso et al., 2016; Ge et al., 2016; Park et al., 2017). 


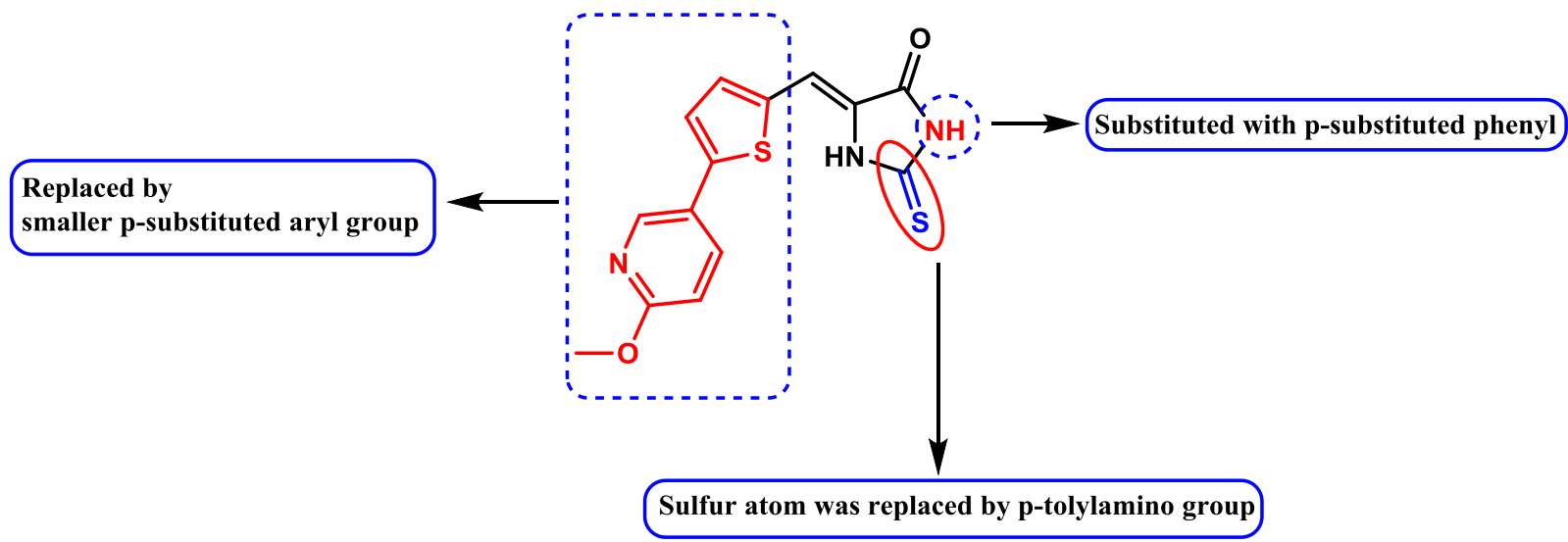

Fig. 2: Strategy for the design of novel 4-imidazolones.

Herein, we designed and synthesized 5-arylidene-3(substituted phenyl)-2-( $p$-tolylamino)-3,5-dihydro-4H-imidazol-4ones based on compounds (I) and (II) through modification of the structure of compound (I) by adding different $p$-substituted arylidene groups at position 5 through knoevenagel condensation reaction with different $P$-substituted benzaldehydeand $p$ substituted phenyl at position 3 instead of $\mathrm{H}$ in compound (I) and different from compound (II) also, the sulfur atom was replaced by $p$-tolylamino group through nucleophilic substitution reaction with $P$-toluidine; to study the impact of these modifications on the cytotoxicity and binding interactions with topo I, (Figure 2). These synthesized 4-imidazolones 9a-f were evaluated for their cytotoxic activity against prostate cancer (PC-3) and breast cancer (MCF-7) cell lines along with a non-cancerous lung fibroblast cell line (WI38). In an attempt to develop a new scaffold for topo I inhibition, we applied molecular docking against human topo I (PDB: 1SC7) to compare the binding interactions of the synthesized compounds versus compounds (I) and (VI) which have been reported to possess topo I inhibitory activity.

\section{MATERIALS AND METHODS}

\section{Instrument and reagents}

Melting points were determined on a Thomas-Hoover capillary apparatus and are uncorrected. Infrared (IR) spectra were carried out on BRUKER Vector 22 (Japan), infrared spectrophotometers and were expressed in wavenumber $\left(\mathrm{cm}^{-1}\right)$ using $\mathrm{KBr}$ disc, at the micro analytical center, Faculty of Science, Cairo University. ${ }^{1} \mathrm{H}$ NMR and ${ }^{13} \mathrm{C}$ NMR spectra were measured on Bruker Advance III $400 \mathrm{MHz}$ for ${ }^{1} \mathrm{H}$ and $100 \mathrm{MHz}$ for ${ }^{13} \mathrm{C}$ (Bruker AG, Switzerland) with BBFO Smart Probe and Bruker 400 AEON Nitrogen-Free Magnet. Data were analyzed using Topspin 3.1 Software, Faculty of Science, Kafrelsheikh University, Egypt in $\mathrm{CDCl}_{3}$ using tetramethylsilane (TMS) as an internal standard, where $J$ (coupling constant) values are estimated in Hertz $(\mathrm{Hz})$ and chemical shifts $\delta$ were reported in ppm units. Mass spectra were carried out on Fennigan MAT, SSQ 7000, Mass spectrometer at the micro analytical center, Faculty of Science, Cairo University, using the ESI ionization mode. Elemental analyses $(\mathrm{C}, \mathrm{H}, \mathrm{N})$ were performed using elemental analyzer Model: Euro EA 3000 (Italy) at the regional center for mycology and biotechnology, Al-Azhar University, Egypt and all compounds were within $\pm 0.4 \%$ from the theoretical values. Column chromatography was performed using Sigma-Aldrich silica gel with high purity grade, Pore size $60 \mathrm{~A}^{\circ}, 70-230$ mesh and 63-200 $\mu \mathrm{m}$. The purity of compounds was confirmed by thin layer chromatography performed with Merck silica Gel 60F254 aluminum sheets. Flash Chromatography was conducted by puriFlash ${ }^{\circledR} 4125$ for flash chromatography (interchem, France) at Nawah Center, Cairo, Egypt. Puriflash flash chromatography system; gradient elution of Hexane: ethyl acetate was used as system, using 80 gm column of normal phase silica and 34 $\mathrm{ml} / \mathrm{min}$ as flow rate, fractions were collected based on photodiode array (PDA) detection and TLC. All other reagents, purchased from the Aldrich Chemical Company (Milwaukee, WI), were used without further purification.

\section{Synthesis}

General procedure for the synthesis of 2-thioxo-3-(substituted phenyl)-imidazolidin-4-ones $3 a-b$

To a solution of $\mathrm{KOH}(11 \mathrm{mmol}, 0.61 \mathrm{~g})$ in water $(50$ $\mathrm{mL})$ and ethanol $(25 \mathrm{~mL})$, glycine $(\mathbf{1}, 10 \mathrm{mmol}, 0.75 \mathrm{~g})$ was added and dissolved. The appropriate arylisothiocyante $(\mathbf{2 a - b}, 11 \mathrm{mmol})$ was added and the reaction mixture was heated under reflux for 3 $\mathrm{h}$, then conc. $\mathrm{HCl}(10 \mathrm{~mL})$ was added and the reflux was continued for another $2 \mathrm{~h}$. The precipitated solid was filtered, dried \& recrystallized from ethanol to give pure compounds $\mathbf{3 a - b}$.

\section{2-thioxo-3-(p-tolyl)imidazolidin-4-one (3a)}

Yellow solid; Yield, 88\%; mp 241-243 ${ }^{\circ} \mathrm{C}$; ${ }^{1} \mathrm{H}$ NMR $\left(\mathrm{CDCl}_{3}\right) \delta 2.44$ (s, 3H, tolyl- $\left.\mathrm{CH}_{3}\right), 4.29\left(\mathrm{~s}, 2 \mathrm{H}, \mathrm{CH}_{2}\right), 7.22(\mathrm{~d}, 2 \mathrm{H}$, $J=7.6 \mathrm{~Hz}$, tolyl H-3, H-5), 7.29 (s, 1H, NH, $\mathrm{D}_{2} \mathrm{O}$ exchangeable), 7.34 (d, 2H, $J=6.4 \mathrm{~Hz}$, tolyl H-2, H-6).

General procedure for the synthesis of 5-arylidene-2-thioxo-3(substituted phenyl)- imidazolidin-4-ones 5a-f

To a mixture of sodium acetate anhydrous $(34 \mathrm{mmol}, 2.8$ g) and $15 \mathrm{ml}$ glacial acetic acid, 3a-b $(10 \mathrm{mmol})$ and the 
appropriate $P$-substituted benzaldehyde (4a-c, $12 \mathrm{mmol})$ were added. The reaction mixture was heated under reflux for $5 \mathrm{~h}$ and the reaction progress was monitored with TLC every $1 \mathrm{~h}$. The reaction mixture was poured into cold water and the obtained solid was filtered, washed with water and ethanol, dried and recrystallized from ethanol to give the pure compounds $\mathbf{5 a - f}$.

\section{5-(4-chlorobenzylidene)-2-thioxo-3-(p-tolyl)imidazolidin-4-one} (5a)

Yellow solid; Yield, 90\%; mp 248-250 ${ }^{\circ} \mathrm{C}$; ${ }^{1} \mathrm{H}$ NMR $\left(\mathrm{CDCl}_{3}\right) \delta 2.45\left(\mathrm{~s}, 3 \mathrm{H}\right.$, tolyl- $\left.\mathrm{CH}_{3}\right), 6.79(\mathrm{~s}, 1 \mathrm{H}$, vinyl $\mathrm{CH}), 7.28(\mathrm{~s}$, 2H, tolyl H-3, H-5), 7.36 (d, 2H, $J=6 \mathrm{~Hz}$, tolyl H-2, H-6) 7.46 (s, $4 \mathrm{H}$, benzylidene H-2, H-3, H-5, H-6), 8.93 (s, 1H, NH, $\mathrm{D}_{2} \mathrm{O}$ exchangeable).

General procedure for the synthesis of 5-arylidene-2-methylthio3-(substituted phenyl)-3,5-dihydro-4H-imidazol-4-ones 7a-f

To a mixture of $\mathrm{KOH}(11 \mathrm{mmol}, 0.61 \mathrm{~g})$ and $15 \mathrm{ml}$ water, compounds (5a-f, $10 \mathrm{mmol}$ ) were added and suspended, 50 $\mathrm{ml}$ methanol was added and the mixture was stirred till became clear then, methyl iodide $(6,12 \mathrm{mmol}, 0.75 \mathrm{~mL})$ was added and the solution was stirred at room temperature for $6 \mathrm{~h}$ then water was added. The obtained solid was filtered, washed with water, dried and recrystallized from ethanol to give the pure compounds $7 \mathbf{a}-\mathbf{f}$.

\section{5-(4-chlorobenzylidene)-2-(methylthio)-3-(p-tolyl)-3,5-dihydro- 4H-imidazol-4-one (7a)}

yellow solid; Yield, 94\%; mp 209-211 ${ }^{\circ} \mathrm{C}$; ${ }^{1} \mathrm{H}$ NMR $\left(\mathrm{CDCl}_{3}\right) 2.44$ (s, 3H, tolyl-CH $\left.\mathrm{CH}_{3}\right), 2.71$ (s, 3H, S-CH $), 6.99$ (s, 1H, vinyl $\mathrm{CH}$ ), 7.23 (d, 2H, $J=8 \mathrm{~Hz}$, tolyl $\mathrm{H}-3, \mathrm{H}-5), 7.32$ (d, 2H, $J=$ $7.6 \mathrm{~Hz}$, tolyl H-2, H-6), 7.42 (d, 2H, $J=8.4 \mathrm{~Hz}$, benzylidene $\mathrm{H}-3$, $\mathrm{H}-5), 8.16$ (d, 2H, $J=8.4 \mathrm{~Hz}$, benzylidene $\mathrm{H}-2, \mathrm{H}-6) ;{ }^{13} \mathrm{C}$ NMR $\left(\mathrm{CDCl}_{3}\right) \delta 13.35,21.29,122.57,127.11,127.84,128.95,129.70$, $130.42,133.03,133.06,135.69,138.50,139.44$.

\section{5-benzylidene-2-(methylthio)-3-(p-tolyl)-3,5-dihydro-4H- imidazol-4-one (7b)}

yellow solid; Yield, 92\%; mp 154-156 ${ }^{\circ} \mathrm{C} ;{ }^{1} \mathrm{H}$ NMR $\left(\mathrm{CDCl}_{3}\right) 2.44$ (s, 3H, tolyl- $\left.\mathrm{CH}_{3}\right), 2.73$ (s, 3H, S-CH$), 7.07$ (s, 1H, vinyl $\mathrm{CH}$ ), 7.25 (d, 2H, $J=8 \mathrm{~Hz}$, tolyl H-3, H-5), 7.33 (d, 2H, $J=$ $8 \mathrm{~Hz}$, tolyl H-2, H-6), 7.41 (t, $1 \mathrm{H}, J=7 \mathrm{~Hz}$, benzylidene $\mathrm{H}-4), 7.47$ (t, $2 \mathrm{H}, J=7.2 \mathrm{~Hz}$, benzylidene $\mathrm{H}-3, \mathrm{H}-5$ ), 8.23 (d, 2H, $J=7.6 \mathrm{~Hz}$, benzylidene $\mathrm{H}-2, \mathrm{H}-6)$.

\section{5-(4-methylbenzylidene)-2-(methylthio)-3-(p-tolyl)-3,5-dihydro- 4H-imidazol-4-one (7c)}

yellow solid; Yield, 96\%; mp 164-166 ${ }^{\circ} \mathrm{C} ;{ }^{1} \mathrm{H}$ NMR $\left(\mathrm{CDCl}_{3}\right) \delta 2.44\left(\mathrm{~s}, 6 \mathrm{H}\right.$, tolyl- $\mathrm{CH}_{3}$ and benzylidene- $\left.\mathrm{CH}_{3}\right), 2.72(\mathrm{~s}$, $\left.3 \mathrm{H}, \mathrm{S}-\mathrm{CH}_{3}\right), 7.07$ (s, $1 \mathrm{H}$, vinyl $\left.\mathrm{CH}\right), 7.24(\mathrm{~d}, 2 \mathrm{H}, J=8.4 \mathrm{~Hz}$, tolyl H-3, H-5), 7.28 (d, 2H, $J=8$ Hz, benzylidene H-3, H-5), 7.32 (d, $2 \mathrm{H}, J=8.4 \mathrm{~Hz}$, tolyl H-2, H-6), 8.13 (d, $2 \mathrm{H}, J=8 \mathrm{~Hz}$, benzylidene H-2, H-6). 3-(4-bromophenyl)-5-(4-chlorobenzylidene)-2-(methylthio)-3,5dihydro-4H-imidazol-4-one (7d)

yellow solid; Yield, 93\%; mp 232-234 ${ }^{\circ} \mathrm{C} ;{ }^{1} \mathrm{H}$ NMR $\left(\mathrm{CDCl}_{3}\right) \delta 2.63\left(\mathrm{~s}, 3 \mathrm{H}, \mathrm{S}-\mathrm{CH}_{3}\right), 6.89(\mathrm{~s}, 1 \mathrm{H}$, vinyl $\mathrm{CH}), 7.16(\mathrm{~d}$, $2 \mathrm{H}, J=8 \mathrm{~Hz}$, phenyl H-3, H-5), 7.32 (d, 2H, $J=8 \mathrm{~Hz}$, benzylidene H-3, H-5), 7.55 (d, 2H, J = 7.6 Hz, phenyl H-2, H-6), 8.05 (d, 2H, $J=8 \mathrm{~Hz}$, benzylidene $\mathrm{H}-2, \mathrm{H}-6)$.

\section{5-benzylidene-3-(4-bromophenyl)-2-(methylthio)-3,5-dihydro- 4H-imidazol-4-one (7e)}

yellow solid; Yield, 90\%; mp 182-184 ${ }^{\circ} \mathrm{C} ;{ }^{1} \mathrm{H}$ NMR $\left(\mathrm{CDCl}_{3}\right) \delta 2.74\left(\mathrm{~s}, 3 \mathrm{H}, \mathrm{S}-\mathrm{CH}_{3}\right), 7.07(\mathrm{~s}, 1 \mathrm{H}$, vinyl $\mathrm{CH}), 7.27(\mathrm{~d}$, $2 \mathrm{H}, J=8.4 \mathrm{~Hz}$, phenyl $\mathrm{H}-3, \mathrm{H}-5), 7.42(\mathrm{t}, 1 \mathrm{H}, J=7 \mathrm{~Hz}$, benzylidene $\mathrm{H}-4), 7.47$ (t, $2 \mathrm{H}, J=7.2 \mathrm{~Hz}$, benzylidene $\mathrm{H}-3, \mathrm{H}-5$ ), 7.65 (d, 2H, $J=8.4 \mathrm{~Hz}$, phenyl H-2, H-6), 8.22 (d, 2H, $J=7.6 \mathrm{~Hz}$, benzylidene $\mathrm{H}-2, \mathrm{H}-6)$.

\section{3-(4-bromophenyl)-5-(4-methylbenzylidene)-2-(methylthio)-3,5- dihydro-4H-imidazol-4-one (7f)}

yellow solid; Yield, 92\%; mp 218-220 ${ }^{\circ} \mathrm{C}$; ${ }^{1} \mathrm{H}$ NMR $\left(\mathrm{CDCl}_{3}\right) \delta 2.32\left(\mathrm{~s}, 3 \mathrm{H}\right.$, benzylidene- $\left.\mathrm{CH}_{3}\right), 2.63\left(\mathrm{~s}, 3 \mathrm{H}, \mathrm{S}-\mathrm{CH}_{3}\right)$, 6.95 (s, $1 \mathrm{H}$, vinyl $\mathrm{CH}), 7.15$ (t, $4 \mathrm{H}, J=8.2 \mathrm{~Hz}$, benzylidene $\mathrm{H}-3$, H-5 and phenyl H-3, H-5), 7.53 (d, 2H, $J=8.4 \mathrm{~Hz}$, phenyl H-2, H6), 8.01 (d, 2H, $J=7.6 \mathrm{~Hz}$, benzylidene $\mathrm{H}-2, \mathrm{H}-6)$.

General procedure for the synthesis of 5-arylidene-3-(substituted phenyl)-2-(p-tolylamino)-3,5-dihydro-4H-imidazol-4-ones 9a-f

To $30 \mathrm{ml}$ glacial acetic acid, compounds (7a-f, $10 \mathrm{mmol})$ and $P$-toluidine $(\mathbf{8}, 12 \mathrm{mmol}, 1.28 \mathrm{~g}$ ) were added. The reaction mixture was heated under reflux for $12 \mathrm{~h}$ and the reaction progress was monitored with TLC every $1 \mathrm{~h}$. The reaction mixture was concentrated and left overnight. The precipitated solid was filtered, washed with ethanol, dried and purified by recrystallization from ethanol9a-c, column chromatography (9d) and flash column chromatography $(\mathbf{9 e}, \mathbf{9 f})$, to give the pure compounds $\mathbf{9 a - f}$.

\section{5-(4-chlorobenzylidene)-3-(p-tolyl)-2-(p-tolylamino)-3,5-dihydro- 4H-imidazol-4-one (9a)}

Yellow solid; Yield, 75\%; mp 241-243 ${ }^{\circ} \mathrm{C}$; IR (KBr) $3293(\mathrm{NH}), 1703(\mathrm{C}=\mathrm{O}), 1651 \quad(\mathrm{C}=\mathrm{C}$ aliphatic $), 1551 \quad(\mathrm{C}=\mathrm{C}$ aromatic) $\mathrm{cm}^{-1} ;{ }^{1} \mathrm{H} \mathrm{NMR}\left(\mathrm{CDCl}_{3}\right) \delta 2.38\left(\mathrm{~s}, 3 \mathrm{H}\right.$, tolylamino- $\left.\mathrm{CH}_{3}\right)$, 2.47 (s, 3H, tolyl- $\left.\mathrm{CH}_{3}\right), 6.39$ (s, $1 \mathrm{H}, \mathrm{NH}, \mathrm{D}_{2} \mathrm{O}$ exchangeable), 6.87 (s, 1H, vinyl $\mathrm{CH}), 7.21(\mathrm{~d}, 2 \mathrm{H}, J=8 \mathrm{~Hz}$, tolylamino $\mathrm{H}-3, \mathrm{H}-5)$, 7.27 (d, 2H, $J=7.6 \mathrm{~Hz}$, tolyl H-3, H-5), $7.41(\mathrm{t}, 4 \mathrm{H}, J=8 \mathrm{~Hz}$, tolylamino H-2, H-6 and tolyl H-2, H-6), $7.56(\mathrm{~d}, 2 \mathrm{H}, J=6.8 \mathrm{~Hz}$, benzylidene $\mathrm{H}-3, \mathrm{H}-5), 8.11(\mathrm{~d}, 2 \mathrm{H}, J=7.6 \mathrm{~Hz}$, benzylidene $\mathrm{H}-2$, $\mathrm{H}-6) ;{ }^{13} \mathrm{C} \mathrm{NMR}\left(\mathrm{CDCl}_{3}\right) \delta 20.87,21.31,117.91,119.76,127.53$, $128.62,128.80,129.70,131.13,132.13,133.80,133.98,134.29$, 134.55,138.65, 140.08,153.09, 168.45; EIMS (m/z) $401.28\left(\mathrm{M}^{+}\right.$, $100 \%), 403.29\left(\mathrm{M}^{+2}, 32.07 \%\right)$. Anal. Calcd for $\mathrm{C}_{24} \mathrm{H}_{20} \mathrm{ClN}_{3} \mathrm{O}: \mathrm{C}$, 71.73; H, 5.02; N, 10.46. Found: C, 71.56; H, 5.17; N, 10.80. 


\section{5-benzylidene-3-(p-tolyl)-2-(p-tolylamino)-3,5-dihydro-4H- imidazol-4-one $(9 b)$}

Yellow solid; Yield, 80\%; mp 208-210 ${ }^{\circ} \mathrm{C}$; IR (KBr) 3090 ( $\mathrm{CH}$ aromatic), $3273(\mathrm{NH}), 1706(\mathrm{C}=\mathrm{O}), 1651 \quad(\mathrm{C}=\mathrm{C}$ aliphatic), $1557\left(\mathrm{C}=\mathrm{C}\right.$ aromatic) $\mathrm{cm}^{-1} ;{ }^{1} \mathrm{H}$ NMR $\left(\mathrm{CDCl}_{3}\right) \delta 2.38(\mathrm{~s}$, $3 \mathrm{H}$, tolylamino- $\left.\mathrm{CH}_{3}\right), 2.46\left(\mathrm{~s}, 3 \mathrm{H}\right.$, tolyl- $\left.\mathrm{CH}_{3}\right), 6.40(\mathrm{~s}, 1 \mathrm{H}, \mathrm{NH}$, $\mathrm{D}_{2} \mathrm{O}$ exchangeable), 6.95 (s, $1 \mathrm{H}$, vinyl $\left.\mathrm{CH}\right), 7.22(\mathrm{~d}, 2 \mathrm{H}, J=8.4$ $\mathrm{Hz}$, tolylamino H-3, H-5), 7.27 (d, 2H, $J=8.8 \mathrm{~Hz}$, tolyl H-3, H-5), 7.38 (q, 3H, $J=7.9 \mathrm{~Hz}$, benzylidene H-3, H-4, H-5), 7.47 (t, 2H, $J$ $=7.6 \mathrm{~Hz}$, tolylamino H-2, H-6), $7.60(\mathrm{~d}, 2 \mathrm{H}, J=8 \mathrm{~Hz}$, tolyl H-2, H-6), 8.19 (d, 2H, $J=7.6 \mathrm{~Hz}$, benzylidene $\mathrm{H}-2, \mathrm{H}-6) ;{ }^{13} \mathrm{C}$ NMR $\left(\mathrm{CDCl}_{3}\right) \delta 20.87,21.31,119.61,127.55,128.58,128.65,128.75$, 129.67, 131.09, 133.70, 134.77, 135.27, 138.28, 139.98, 152.82, 168.66; EIMS (m/z) $367.33\left(\mathrm{M}^{+}, 84.11 \%\right), 368.32\left(\mathrm{M}^{+1}, 100 \%\right)$. Anal. Calcd for $\mathrm{C}_{24} \mathrm{H}_{21} \mathrm{~N}_{3} \mathrm{O}: \mathrm{C}, 78.45 ; \mathrm{H}, 5.76 ; \mathrm{N}, 11.44$. Found: $\mathrm{C}$, 78.68; H, 5.81; N, 11.61 .

\section{5-(4-methylbenzylidene)-3-(p-tolyl)-2-(p-tolylamino)-3,5-dihydro- 4H-imidazol-4-one (9c)}

Yellow solid; Yield, 70\%; mp 238-240 ${ }^{\circ} \mathrm{C}$; IR (KBr) $3284(\mathrm{NH}), 1699(\mathrm{C}=\mathrm{O}), 1649$ (C=C aliphatic), $1551 \quad(\mathrm{C}=\mathrm{C}$ aromatic) $\mathrm{cm}^{-1} ;{ }^{1} \mathrm{H}$ NMR $\left(\mathrm{CDCl}_{3}\right) \delta 2.38\left(\mathrm{~s}, 3 \mathrm{H}\right.$, tolylamino- $\left.\mathrm{CH}_{3}\right)$, $2.43\left(\mathrm{~s}, 3 \mathrm{H}\right.$, tolyl- $\left.\mathrm{CH}_{3}\right), 2.47\left(\mathrm{~s}, 3 \mathrm{H}\right.$, benzylidene- $\left.\mathrm{CH}_{3}\right) 6.31(\mathrm{~s}, 1 \mathrm{H}$, $\mathrm{NH}, \mathrm{D}_{2} \mathrm{O}$ exchangeable), 6.95 (s, $1 \mathrm{H}$, vinyl $\left.\mathrm{CH}\right), 7.21(\mathrm{~d}, 2 \mathrm{H}, J=8$ $\mathrm{Hz}$, tolylamino H-3, H-5), 7.28 (d, d, 4H, $J=2,1.6 \mathrm{~Hz}$, tolyl H-3, $\mathrm{H}-5$ and benzylidene $\mathrm{H}-3, \mathrm{H}-5), 7.40$ (d, 2H, $J=8 \mathrm{~Hz}$, tolylamino H-2, H-6), 7.61 (d, 2H, $J=8.4 \mathrm{~Hz}$, tolyl H-2, H-6), 8.1 (d, 2H, $J=$ $8 \mathrm{~Hz}$, benzylidene $\mathrm{H}-2, \mathrm{H}-6) ;{ }^{13} \mathrm{C} \mathrm{NMR}\left(\mathrm{CDCl}_{3}\right) \delta 20.86,21.30$, $21.60,119.53,119.96,127.55,128.84,129.39$, 129.63, 131.08, $132.49,133.55,134.88,137.54,138.94,139.89,152.41,168.67$; EIMS (m/z) $381.21\left(\mathrm{M}^{+}, 9.64 \%\right), 382.33\left(\mathrm{M}^{+1}, 1.81 \%\right), 43.96$ (100\%). Anal. Calcd for $\mathrm{C}_{25} \mathrm{H}_{23} \mathrm{~N}_{3} \mathrm{O}$ : C, 78.71; H, 6.08; N, 11.02. Found: C, 78.84; H, 5.98; N, 11.21.

\section{3-(4-bromophenyl)-5-(4-chlorobenzylidene)-2-(p-tolylamino)- 3,5-dihydro-4H-imidazol-4-one (9d)}

Yellow solid; Yield, 75\%; mp 204-206 ${ }^{\circ} \mathrm{C}$; IR (KBr) $3293(\mathrm{NH}), 1705(\mathrm{C}=\mathrm{O}), 1650 \quad(\mathrm{C}=\mathrm{C}$ aliphatic), $1554 \quad(\mathrm{C}=\mathrm{C}$ aromatic) $\mathrm{cm}^{-1} ;{ }^{1} \mathrm{H}$ NMR $\left(\mathrm{CDCl}_{3}\right) \delta 2.39\left(\mathrm{~s}, 3 \mathrm{H}\right.$, tolyl- $\left.\mathrm{CH}_{3}\right), 6.31$ (s, 1H, NH, $\mathrm{D}_{2} \mathrm{O}$ exchangeable), $6.86(\mathrm{~s}, 1 \mathrm{H}$, vinyl $\mathrm{CH}), 7.22(\mathrm{~d}$, $2 \mathrm{H}, J=8.4 \mathrm{~Hz}$, tolylamino H-3, H-5), $7.28(\mathrm{t}, 2 \mathrm{H}, J=4.2 \mathrm{~Hz}$, phenyl H-3, H-5), 7.41 (d, 2H, $J=8.4 \mathrm{~Hz}$, benzylidene H-3, H-5), 7.56 (d, 2H, $J=8.4 \mathrm{~Hz}$, tolylamino H-2, H-6), 7.74 (d, 2H, $J=8.4$ $\mathrm{Hz}$, phenyl H-2, H-6), 8.1 (d, 2H, $J=8.4 \mathrm{~Hz}$, benzylidene $\mathrm{H}-2, \mathrm{H}-$ $6) ;{ }^{13} \mathrm{C} \mathrm{NMR}\left(\mathrm{CDCl}_{3}\right) \delta 20.88,118.58,119.79,123.83,129.24$, $129.76,130.47,130.89,132.22$, 133.62, 133.77, 134.19, 134.41, 134.51, 138.34, 152.28, 168.15; EIMS (m/z) $466.59\left(\mathrm{M}^{+}, 8.21 \%\right)$, $431.33(100 \%)$. Anal. Calcd for $\mathrm{C}_{23} \mathrm{H}_{17} \mathrm{BrClN}_{3} \mathrm{O}: \mathrm{C}, 59.18 ; \mathrm{H}$, 3.67 ; N, 9.00. Found: C, 58.93; H, 3.70; N, 9.21.

\section{5-benzylidene-3-(4-bromophenyl)-2-(p-tolylamino)-3,5-dihydro- 4H-imidazol-4-one (9e)}

Yellow solid; Yield, 73\%; mp 186-188 ${ }^{\circ} \mathrm{C}$; IR (KBr) $3274(\mathrm{NH}), 1707(\mathrm{C}=\mathrm{O}), 1649(\mathrm{C}=\mathrm{C}$ aliphatic $), 1556 \quad(\mathrm{C}=\mathrm{C}$ aromatic) $\mathrm{cm}^{-1} ;{ }^{1} \mathrm{H} \mathrm{NMR}\left(\mathrm{CDCl}_{3}\right) \delta 2.38\left(\mathrm{~s}, 3 \mathrm{H}\right.$, tolylamino- $\left.\mathrm{CH}_{3}\right)$, $6.39\left(\mathrm{~s}, 1 \mathrm{H}, \mathrm{NH}, \mathrm{D}_{2} \mathrm{O}\right.$ exchangeable), $6.94(\mathrm{~s}, 1 \mathrm{H}$, vinyl $\mathrm{CH}), 7.22$ (d, $2 \mathrm{H}, J=8.4 \mathrm{~Hz}$, tolylamino H-3, H-5), 7.27 (d, 2H, $J=6.8 \mathrm{~Hz}$, phenyl H-3, H-5), 7.37 (t, 1H, $J=7.2 \mathrm{~Hz}$, benzylidene $\mathrm{H}-4), 7.46$ (t, 2H, $J=7.4 \mathrm{~Hz}$, benzylidene $\mathrm{H}-3, \mathrm{H}-5), 7.59$ (d, $2 \mathrm{H}, J=6.8 \mathrm{~Hz}$, phenyl H-2, H-6), 7.72 (d, 2H, $J=8 \mathrm{~Hz}$, tolylamino $\mathrm{H}-2, \mathrm{H}-6)$, $8.17(\mathrm{~d}, 2 \mathrm{H}, J=6.8 \mathrm{~Hz}$, benzylidene $\mathrm{H}-2, \mathrm{H}-6) ;{ }^{13} \mathrm{C} \mathrm{NMR}\left(\mathrm{CDCl}_{3}\right)$ $\delta 20.88,119.78,120.30,123.71,128.62,128.87,129.25,129.74$, $130.62,131.17,133.69,133.99,134.54,135.01,137.65,152.02$, 168.23; EIMS (m/z) $431.26\left(\mathrm{M}^{+}, 100 \%\right), 433.25\left(\mathrm{M}^{+2}, 31.18 \%\right)$. Anal. Calcd for $\mathrm{C}_{23} \mathrm{H}_{18} \mathrm{BrN}_{3} \mathrm{O}$ : C, 63.90; H, 4.20; N, 9.72. Found: C, 64.18; H, 4.31; N, 9.91.

\section{3-(4-bromophenyl)-5-(4-methylbenzylidene)-2-(p-tolylamino)- 3,5-dihydro-4H-imidazol-4-one (9f)}

Yellow solid; Yield, 70\%; mp 176-178 ${ }^{\circ} \mathrm{C}$; IR (KBr) $3284(\mathrm{NH}), 1698(\mathrm{C}=\mathrm{O}), 1648(\mathrm{C}=\mathrm{C}$ aliphatic), $1547 \quad(\mathrm{C}=\mathrm{C}$ aromatic) $\mathrm{cm}^{-1} ;{ }^{1} \mathrm{H} \mathrm{NMR}\left(\mathrm{CDCl}_{3}\right) \delta 2.38\left(\mathrm{~s}, 3 \mathrm{H}\right.$, tolylamino- $\left.\mathrm{CH}_{3}\right)$, $2.43\left(\mathrm{~s}, \quad 3 \mathrm{H}\right.$, benzylidene- $\left.\mathrm{CH}_{3}\right), 6.36\left(\mathrm{~s}, \quad 1 \mathrm{H}, \quad \mathrm{NH}, \quad \mathrm{D}_{2} \mathrm{O}\right.$ exchangeable), 6.95 (s, 1H, vinyl CH) $7.24(\mathrm{~d}, \mathrm{~s}, 6 \mathrm{H}, J=7.6 \mathrm{~Hz}$, tolylamino $\mathrm{H}-3, \mathrm{H}-5$, benzylidene $\mathrm{H}-3, \mathrm{H}-5$ and phenyl $\mathrm{H}-3, \mathrm{H}-5)$, 7.59 (d, 2H, $J=4.8 \mathrm{~Hz}$, tolylamino H-2, H-6), 7.72 (d, 2H, J= 7.2 $\mathrm{Hz}$, phenyl H-2, H-6), 8.07 (d, 2H, $J=4 \mathrm{~Hz}$, benzylidene $\mathrm{H}-2, \mathrm{H}-$ $6) ;{ }^{13} \mathrm{C} \mathrm{NMR}\left(\mathrm{CDCl}_{3}\right) \delta 20.86,21.59,119.69,120.69,123.64$, $129.25,129.44,129.71,130.71,131.17,132.24,133.68,133.85$, 134.64, 136.84, 139.24, 151.60, 168.24; EIMS (m/z) $445.26\left(\mathrm{M}^{+}\right.$, $41.28 \%), 447.26\left(\mathrm{M}^{+2}, 2.42 \%\right), 446.29$ (100\%). Anal. Calcd for $\mathrm{C}_{24} \mathrm{H}_{20} \mathrm{BrN}_{3} \mathrm{O}$ : C, 64.58; H, 4.52; N, 9.41. Found: C, 64.71; H, $4.54 ; \mathrm{N}, 9.54$.

\section{In vitro cytotoxicity screening \\ Cell line propagation}

Mammary gland breast cancer cell line (MCF-7), human prostate cancer cell line (PC-3) and human lung fibroblast cell line (WI-38) were obtained from ATCC via holding company for biological products and vaccines (VACSERA), Cairo, Egypt. The reagents RPMI-1640 medium, MTT, DMSO and 5-fluorouracil were purchased from (sigma co., St. Louis, USA). Fetal bovine serum was obtained from (GIBCO, UK). The different cell lines mentioned above were used to determine the inhibitory effects of the tested compounds on cell growth using the MTT assay (Denizot and Lang, 1986).This colorimetric assay is based on the conversion of the yellow tetrazolium bromide (MTT) to a purple formazan derivative by mitochondrial succinate dehydrogenase in viable cells.

\section{MTT assay}

The cells were cultured in RPMI-1640 medium with $10 \%$ fetal bovine serum. Antibiotics were added 100 units $/ \mathrm{ml}$ penicillin and $100 \mu \mathrm{g} / \mathrm{ml}$ streptomycin at $37{ }^{\circ} \mathrm{C}$ in a $5 \% \mathrm{CO}_{2}$ incubator. The cells were seeded in a 96-well plate at a density of $1.0 \times 10^{4}$ cells/well at $37{ }^{\circ} \mathrm{C}$ for $48 \mathrm{~h}$ under $5 \% \mathrm{CO}_{2}$. After incubation, the cells were treated with different concentrations of compounds and incubated for 24 h. $20 \mu \mathrm{L}$ of MTT solution at $5 \mathrm{mg} / \mathrm{ml}$ was added 
and incubated for $4 \mathrm{~h}$. Dimethyl sulfoxide (DMSO) in volume of $100 \mu \mathrm{L}$ was added into each well to dissolve the purple formed formazan. The colorimetric assay was measured and recorded at absorbance of $570 \mathrm{~nm}$ using a plate reader (EXL 800 USA). The relative cell viability in percentage was calculated as (A570 of treated samples/A570 of untreated sample) X 100(Mauceri et al., 1998). Cytotoxic activities of the target compounds were expressed as $\mathrm{IC}_{50}$ (the concentrations of compounds required to produce $50 \%$ inhibition of cell growth). $\mathrm{IC}_{50}$ values were calculated using sigmoidal concentration response curve fitting models by Sigma plot software version 13.0, (Figure 3). The results reported are means of at least three separate experiments. Significant differences were analyzed using one-way ANOVA wherein the differences were considered to be significant at $\mathrm{P}$ $<0.05$.
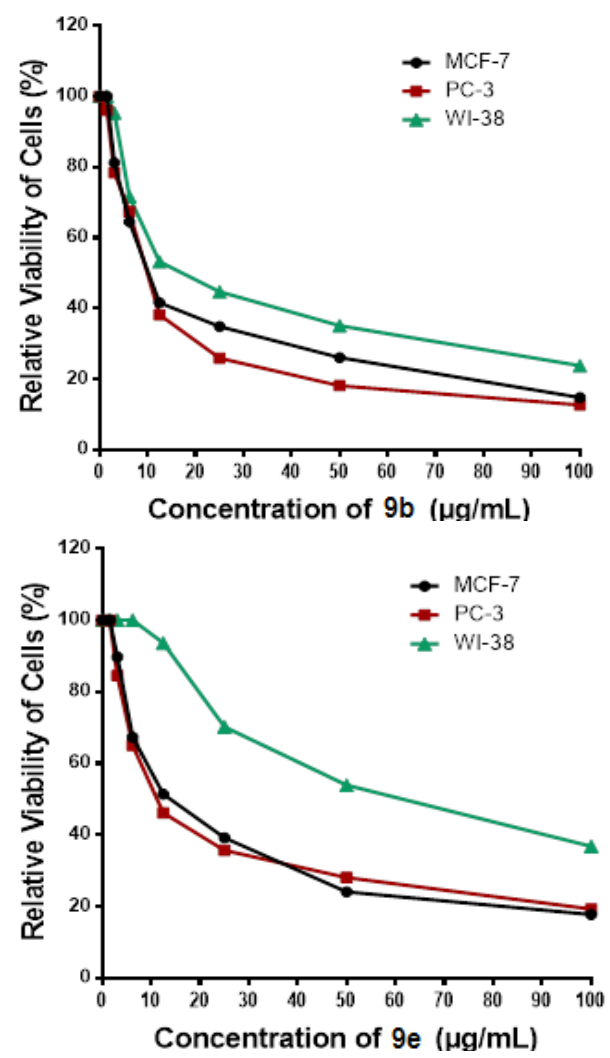

Fig. 3: Cytotoxicity curves of the two most potent compounds $9 \mathrm{~b}$, e against the three cell lines.

\section{Molecular docking study}

Docking for the most potent compounds $\mathbf{9 b , e}$, compound (I) and MJ238 (VI) was conducted via their 3D structure built and energy minimized using MMFF94x of Molecular Operating Environment energy minimization module (MOE, Version 2005.06, Chemical Computing Group Inc., Montreal, Quebec, Canada) (MOE. Chemical Computing Group). (PDB ID: 1SC7) that represents $3 \mathrm{D}$ crystal structure of human topo I was selected from Protein Data Bank database (http://www.rcsb.org). The missing residues and side chains of the crystal structure were repaired, the $3 \mathrm{D}$ protonation of the target was achieved for molecular docking and the energy minimized using MMFF94x 24. The active site for docking was determined as a sphere of all atoms within $6.5 \mathrm{~A}$ radius of the co-crystallized reference ligand in the crystal structure complex of the enzyme. Water molecules were removed from the active site of the target. The tested compounds were loaded individually in mol 2 format into FlexX module in LeadIT 2.1.8 software package (http://www.Biosolveit.De/flexx/). Full score contribution threshold and no score contribution threshold was set as default to 0.30 and 0.70 respectively. The protein ligand clash (maximum allowed overlap volume) and intra ligand clash (clash factor) was set as default to $2.9 \mathrm{~A}^{\circ}$ and $0.6 \mathrm{~A}^{\circ}$, respectively in the docking considering hydrogen in internal clash tests. Stereo mode during docking was considered.

Maximum number of solutions per iteration was set to 200 and maximum number of solutions per fragmentation was set to 200. Top 10 poses were kept. For scoring analysis; the best FlexX score for each compound was taken and compared to the scores of the other compounds (I) and MJ238 (VI). Pose View, algorithm implemented in FlexX software is used to display 2D molecular complexes incorporating a simple, easy-to-perceive arrangement of the ligand and the amino acids towards which it forms interactions (Stierand and Rarey, 2007; Stierand andRarey, 2010).

\section{RESULTS AND DISCUSSIONS}

\section{Chemistry}

The synthesis of 5-arylidene-3-(substituted phenyl)-2-(ptolylamino)-4-imidazolones 9a-f was conducted according to (Scheme 1). 2-thioxo-3-(substituted phenyl)-imidazolidin-4-ones 3a-b were synthesized in good yields via the reaction of glycine $\mathbf{1}$ with the appropriate arylisothiocyanate $\mathbf{2 a}$ or $\mathbf{2 b}$ under Edman degradation like condition. In the presence of $\mathrm{KOH}$, nucleophilic addition of glycine- $\mathrm{NH}_{2}$ on arylisothiocyantehas been occurred followed by condensation reaction by reflux with conc. $\mathrm{HCl}$ yielding the cyclic 3a-b. The ${ }^{1} \mathrm{H}-\mathrm{NMR}$ spectra of $\mathbf{3 a}$ showed the presence of two singlet signals at $2.44 \mathrm{ppm}$ and $4.29 \mathrm{ppm}$ that assigned for tolyl- $\mathrm{CH}_{3}$ in position 3 and aliphatic $\mathrm{CH}_{2}$ in position 5 , respectively. In addition, two doublet signals appeared at 7.22 and $7.34 \mathrm{ppm}$ attributed to the four tolyl hydrogens. Moreover, the singlet signal of NH appeared at $7.29 \mathrm{ppm}$.

Compounds 3a-b were subsequently condensed with different $P$-substituted benzaldehyde 4a-c according to knoevenagel reaction in the presence of anhydrous sodium acetate and glacial acetic acid yielding 5-arylidene-2-thioxo-3-(substituted phenyl)- imidazolidin-4-ones 5a-f (Khodair, 2001). Compound 5a was confirmed by ${ }^{1} \mathrm{H}-\mathrm{NMR}$ though the appearance of a singlet signal at $6.79 \mathrm{ppm}$ attributed to vinyl $\mathrm{CH}$ at position 5 also, signals at 7.28 and $7.36 \mathrm{ppm}$ corresponds to the fourtolyl hydrogens. In addition, the signal at 7.46 is attributed to the four benzylidene hydrogens. Moreover, a singlet exchangeable signal at $8.93 \mathrm{ppm}$ assigned for $\mathrm{NH}$.

Furthermore, 5-arylidene-2-methylthio-3-(substituted phenyl)-3,5-dihydro-4H-imidazol-4-ones 7a-f were synthesized in 


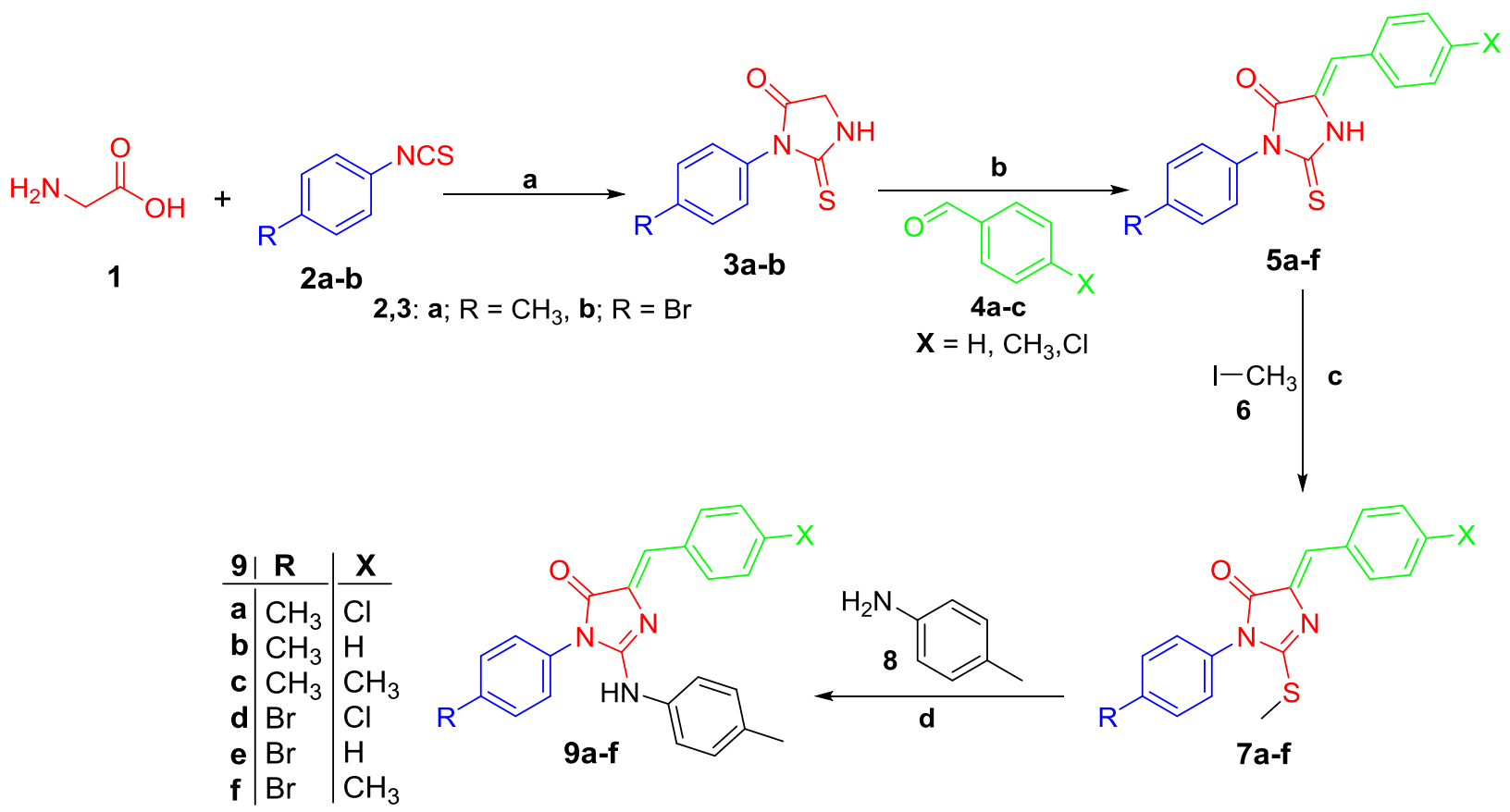

Scheme 1: Reagents and conditions: (a) i) aqueous $\mathrm{KOH}$, reflux, $3 \mathrm{~h}$; ii) Conc. $\mathrm{HCl}$, reflux, $2 \mathrm{~h}$; (b) anhydrous sodium acetate, glacial acetic acid, appropriate $P$-substituted benzaldehyde, reflux, 5 h; c) KOH, MeOH, $\mathrm{CH}_{3} \mathrm{I}, \mathrm{RT}, 6 \mathrm{~h}$; d) $P$-toluidine, glacial acetic acid, reflux, $12 \mathrm{~h}$.

excellent yields via the reaction of $\mathbf{5 a - f}$ in aqueous $\mathrm{KOH}$ and methanol with methyl iodide (6) at room temperature (Khodair et al., 1998). ${ }^{1} \mathrm{H}-\mathrm{NMR}$ spectra confirmed the structures of $\mathbf{7 a - f}$, the dissapearence of the singlet signal of $\mathrm{NH}$ revealed the Smethylation, also the presence of a singlet signal at $2.63-2.74 \mathrm{ppm}$ that assigned for $\mathrm{S}_{-} \mathrm{CH}_{3}$ confirmed the same result. In addition, ${ }^{13} \mathrm{C}-\mathrm{NMR}$ of $7 \mathbf{a}$ revealed the presence of the $\mathrm{S}-\mathrm{CH}_{3}$ signal at 13.35 ppm besides the other signals of the compound.

Finally, the synthesis of 5-arylidene-3-(substituted phenyl)-2-( $p$-tolylamino)-3,5-dihydro-4H-imidazol-4-ones 9a-f were accomplished through nucleophilic substitution reaction by reflux of $\mathbf{7 a - f}$ with $P$-toluidine $(\mathbf{8})$ in glacial acetic acid. The evolution of methanethiol odor showed that the reaction has been occurred. The structures of 9a-f were confirmed by spectral and elemental analysis. IR spectra revealed the appearance of a stretching band at $1547-1557 \mathrm{~cm}^{-1}$ assigned for aromatic $\mathrm{C}=\mathrm{C}$ also, a stretching band at $1648-1651 \mathrm{~cm}^{-1}$ corresponds to aliphatic $\mathrm{C}=\mathrm{C}$. Moreover, the starching band at $3273-3293 \mathrm{~cm}^{-1}$ corresponds to tolylamino $\mathrm{NH}$ and absorption band at $1698-1707 \mathrm{~cm}^{-1}$ attributed to carbonyl group. ${ }^{1} \mathrm{H}-\mathrm{NMR}$ spectra showed the presence of a singlet signal at 2.38-2.39 ppm assigned for tolylamino $\mathrm{CH}_{3}$, a singlet signal at 6.31-6.40 ppm corresponds to the tolylamino $\mathrm{NH}$ and a singlet signal at $6.86-6.6 .95 \mathrm{ppm}$ attributed to vinyl $\mathrm{CH}$ at position 5 also, the tolylamino hydrogens have been appeared at 7.21-7.7.27 ppm for tolylamino $\mathrm{H}-3$ and $\mathrm{H}-5$ and 7.40-7.72 ppm for tolylamino $\mathrm{H}-2$ and $\mathrm{H}-6 .{ }^{13} \mathrm{C}-\mathrm{NMR}$ revealed the presence of signals at 20.86-20.88 ppm, 117.91-119.78 ppm and 168.15$168.67 \mathrm{ppm}$ attributed to tolylamino- $\mathrm{CH}_{3}$, vinyl $\mathrm{CH}$ and carbonyl group, respectively besides the other signals of the compounds. Additionally, the structures of 9a-f were confirmed by Mass spectroscopy and Elemental analysis.

\section{In vitro cytotoxicity screening}

The novel compounds 9a-f were screened for their in vitro cytotoxicity against human prostate cancer cell line (PC-3), human breast cancer cell line (MCF-7) and non-cancerous human lung fibroblast cell line (WI-38) using 5-fluorouracil as a positive control by MTT assay. $\mathrm{IC}_{50}$ values were presented in $\mu \mathrm{mol} / \mathrm{ml}$ and selectivity index (S. I.) values were calculated by dividing $\mathrm{IC}_{50}$ value of the target compound against the normal cell line over that against the cancer cell line (Jung et al., 2012) are presented in (Table 1 and 2). Five compounds $9 \mathbf{b}-\mathbf{f}$ out of six showed potent cytotoxic activities against $\mathrm{PC}-3$ with $\mathrm{IC}_{50}$ range $(28-68 \mathrm{nmol} / \mathrm{ml})$ and all compounds are selective to PC-3. Four compounds $\mathbf{9 b}$, 9d-f possessed potent cytotoxic activities against MCF-7 with $\mathrm{IC}_{50}$ range $(34-82 \mathrm{nmol} / \mathrm{ml})$ and all compounds are selective to MCF-7. While, 5-fluorouracil showed $\mathrm{IC}_{50}=60$ and $40 \mathrm{nmol} / \mathrm{ml}$ against PC-3 and MCF-7, respectively. Compound (9b) is the most potent against both PC-3 and MCF-7 with $\mathrm{IC}_{50}=28$ and $34 \mathrm{nmol} / \mathrm{ml}$ and S.I. $=2.07$ and 1.70, respectively. Furthermore, compound $(\mathbf{9 e})$ is the second most potent against both PC-3 and MCF-7 with $\mathrm{IC}_{50}=$ 33 and $36 \mathrm{nmol} / \mathrm{ml}$ and also is the most selective against both cancer cell lines with S.I. $=4.15$ and 3.80 , respectively. In contrast, Compound (9a) is the least potent and most non-selective for both cancer cell lines.

Regarding both PC-3 and MCF-7 cell lines, the cytotoxicity of compounds bearing $P$-tolyl group at position $39 \mathrm{a}-\mathrm{c}$ is dependent on the $p$-substituent on benzylidene in position 5 which the unsubstituted benzylidene is more favored then $p$ methyl and $p$-chloro benzylidene analogues. Similarly, the cytotoxicity of compounds having $P$-bromophenyl at position 3 9d-f is dependent on the $p$-substituent on benzylidene in position 5 with the same order as 9a-c. In addition, with the exception of 
(9b), the $P$-bromophenyl at position 3 is more favored for cytotoxicity against both PC-3 and MCF-7 cell lines than $p$-tolyl substitution. Apparently, the impact of $P$-substitution on 3benzylidene is more significant on cytotoxicity than that on 3phenyl.

Furthermore, with the exception of $\mathbf{9 b}$, compounds with unsubstituted benzylidene at position 5are more selective for both cancer cell lines then $p$-methyl and $p$-chloro benzylidene analogues. Moreover, $P$-bromophenyl substitution at position 3 is more preferable than $p$-tolyl for selectivity against both cancer cell lines. Interestingly, all the tested compounds $9 \mathbf{a}-\mathbf{f}$ are more potent and selective against prostate cancer cell line (PC-3) than breast cancer cell line (MCF-7).

Table 1: Anti-proliferative activity of target imidazolones 9a-ftowards PC-3 and MCF-7 cancer cell lines.

\begin{tabular}{|c|c|c|c|c|}
\hline \multirow[t]{2}{*}{ Compound } & \multirow[t]{2}{*}{$\mathbf{R}$} & \multirow[t]{2}{*}{$\mathbf{X}$} & \multicolumn{2}{|c|}{$\mathrm{IC}_{50}(\mu \mathrm{M} / \mathrm{mL})^{\mathrm{a}}$} \\
\hline & & & PC-3 & MCF-7 \\
\hline 9a & $\mathrm{CH}_{3}$ & $\mathrm{Cl}$ & $0.112 \pm 2.7$ & $0.136 \pm 2.9$ \\
\hline $9 b$ & $\mathrm{CH}_{3}$ & $\mathrm{H}$ & $0.028 \pm 1.1$ & $0.034 \pm 1.2$ \\
\hline $9 \mathrm{c}$ & $\mathrm{CH}_{3}$ & $\mathrm{CH}_{3}$ & $0.059 \pm 1.8$ & $0.115 \pm 2.6$ \\
\hline 9d & $\mathrm{Br}$ & $\mathrm{Cl}$ & $0.068 \pm 2.2$ & $0.082 \pm 2.3$ \\
\hline $9 e$ & $\mathrm{Br}$ & $\mathrm{H}$ & $0.033 \pm 1.3$ & $0.036 \pm 1.4$ \\
\hline 9f & $\mathrm{Br}$ & $\mathrm{CH}_{3}$ & $0.044 \pm 1.6$ & $0.057 \pm 1.7$ \\
\hline 5-Fluorouracil & - & - & $0.060 \pm 0.45$ & $0.040 \pm 0.34$ \\
\hline
\end{tabular}

${ }^{\mathrm{a}} \mathrm{IC}_{50}$ values are the mean $\pm \mathrm{SD}$ of three separate experiments.

Table 2: Anti-proliferative activity of target imidazolones 9a-fagainst human lung fibroblast cell line (WI-38) and selectivity index regarding PC-3 \& MCF-7.

\begin{tabular}{llll}
\hline \multirow{2}{*}{ Compound } & $\mathbf{I C}_{\mathbf{5 0}}(\boldsymbol{\mu M} \mathbf{M} / \mathbf{m L})^{\mathbf{a}}$ & S. I. & \\
\cline { 2 - 4 } & $\mathbf{W I - 3 8}$ & $\mathbf{P C - 3}$ & MCF-7 \\
\hline 9a & $0.089 \pm 1.9$ & 0.794 & 0.654 \\
9b & $0.058 \pm 1.4$ & 2.071 & 1.70 \\
9c & $0.126 \pm 2.6$ & 2.135 & 1.095 \\
9d & $0.094 \pm 2.2$ & 1.38 & 1.146 \\
9e & $0.137 \pm 3.4$ & 4.151 & 3.80 \\
9f & $0.121 \pm 3.1$ & 2.75 & 2.122 \\
5-Fluorouracil & $0.051 \pm 0.51$ & 0.85 & 1.27
\end{tabular}

${ }^{\mathrm{a}} \mathrm{IC}_{50}$ values are the mean $\pm \mathrm{SD}$ of three separate experiments.

\section{Molecular docking study}

Molecular docking study was accomplished on the most potent two compounds $\mathbf{9 b}$, e into human topoisomerase I (topo I) enzyme using FlexX module in LeadIT 2.1.8 software-package and compared with the docking results of compound (I) and indenoisoquinoline (MJ238, VI) that have been reported to have topo I inhibitory activity in order to interpret the differences in the binding interactions at the molecular level as inhibitors of human topo $\mathrm{I}$ in an attempt to rationalize their molecular mechanism as cytotoxic agents. 3D crystal structure of human topo I (PDB ID: 1SC7) complexed with the reference topo I inhibitor (MJ238, VI) and covalently bonded with 22 base pairs DNA was chosen for our molecular docking study.
From the SAR study of topo I inhibitors, it was found that Asp 533 residue is required for the sensitivity of the enzyme to camptothecin (III) also, the closest enzyme-camptothecin interaction is $2.9 \mathrm{~A}^{\circ}$ from the $\mathrm{N}$ of $\mathrm{Arg} 364$ to $\mathrm{N}$ of ring-B. The $\mathrm{C}$ ring carbonyl of MJ238 is on the minor groove and forms a bidentate interaction with the two nitrogens of Arg 364 residue also, its butyl carboxylic acid substituent of the B ring projects in the major groove toward Asn 352 and Ala 351 residues. Besides Asn 352 and Ala 351, Glu 356, Pro 431, Lys 751 and Asn 722 residues are present at the major groove cavity. In addition, $\mathrm{C}$ and D-rings of MJ 238 stacks on the intact DNA strand similar to rings A and B of camptothecin. The interaction with Arg 364 is common between all three different classes of topo I inhibitors representing the sole interaction on the minor groove (Staker et al., 2005). Also, it was reported that Asn 722 is important for subnuclear dynamics and stabilization of camptothecin-induced topo I cleavage complexes (Das et al., 2016).

From the docking results, both compounds $(9 \mathbf{b})$ and $(\mathbf{9 e})$ showed approximately equal good binding interactions with docking scores (-28.018 and $-28.2490 \mathrm{Kcal} / \mathrm{mol}$, respectively) forming one hydrogen bond better than that of compound (1) that showed docking score $(-24.7890 \mathrm{Kcal} / \mathrm{mol})$ with two hydrogen bonds, (Figure 4-6). Also, (MJ238, VI) exhibited a docking score $(-32.0139 \mathrm{Kcal} / \mathrm{mol})$ with 2 hydrogen bonds, (Figure 7).

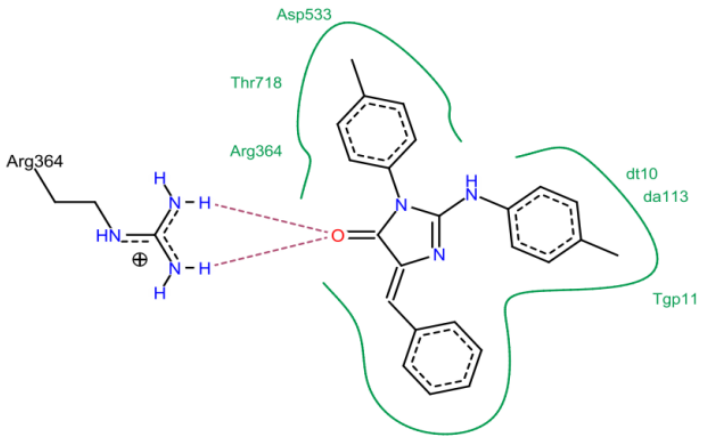

(a)

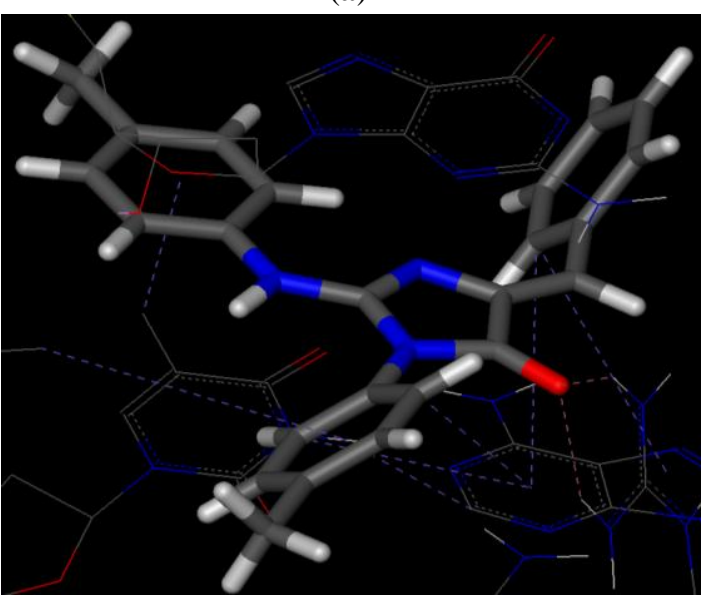

(b)

Fig. 4a, b: 2D and 3D poses view of compound (9b) docked against topo I (PDB: 1SC7). In 2D, red, orange or violet dashed line represents hydrogen bonding while; the green solid line represents hydrophobic interactions. In 3D, red dashed line represents hydrogen bonding while; violet dashed line represents hydrophobic interactions. 

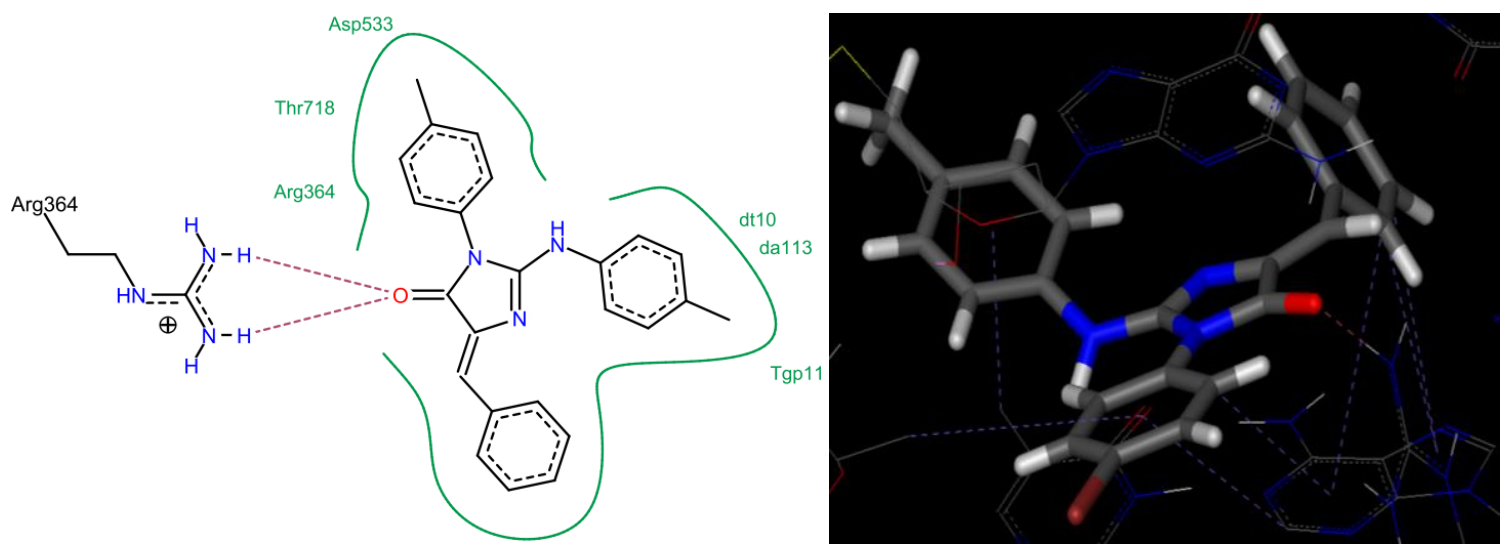

(a)

(b)

Fig. 5a, b: 2D and 3D poses view of compound (9e) docked against topo I (PDB: 1SC7). In 2D, red, orange or violet dashed line represents hydrogen bonding while; the green solid line represents hydrophobic interactions. In 3D, red dashed line represents hydrogen bonding while; violet dashed line represents hydrophobic interactions.

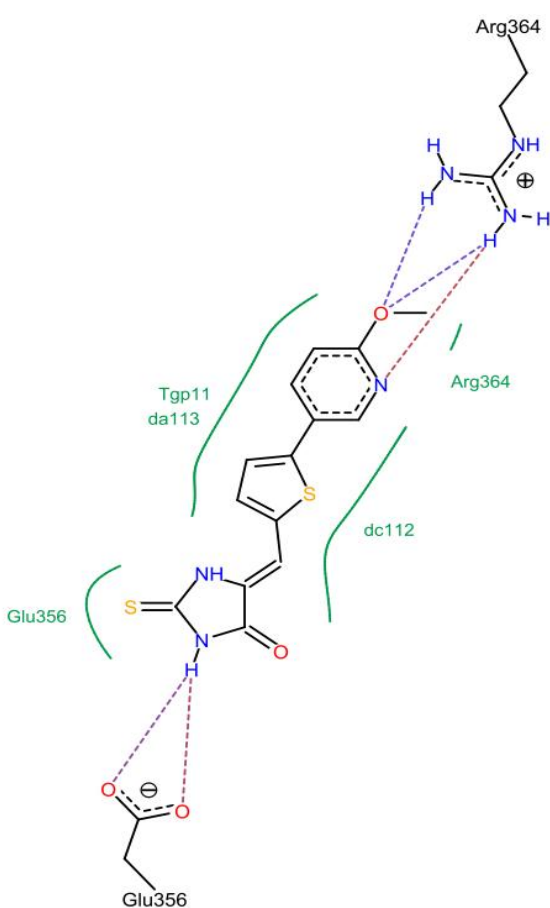

(a)

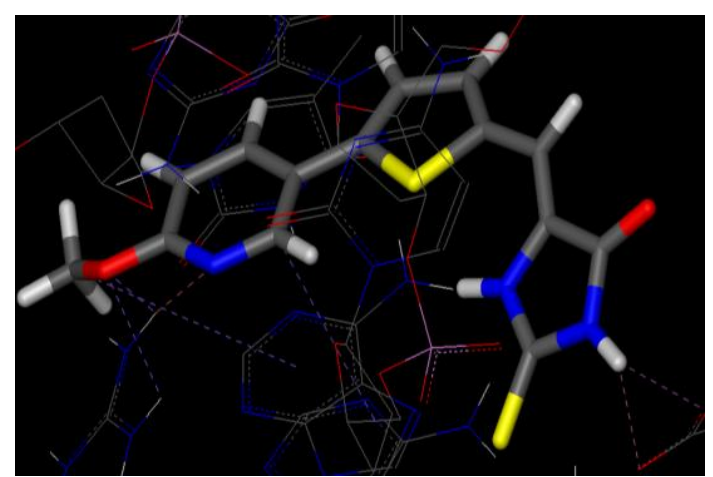

(b)

Fig. 6a, b: 2D and 3D poses view of compound (I) docked against topo I (PDB: 1SC7). In 2D, red, orange or violet dashed line represents hydrogen bonding while; the green solid line represents hydrophobic interactions. In $3 \mathrm{D}$, red dashed line represents hydrogen bonding while; violet dashed line represents hydrophobic interactions.

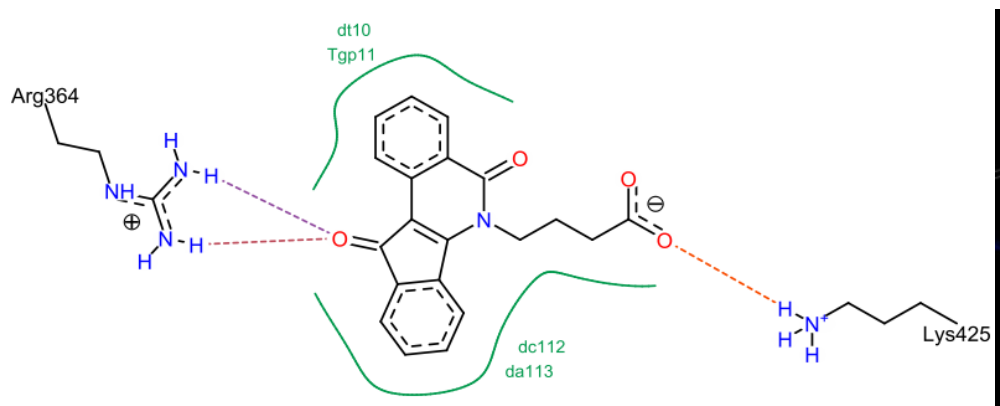

(a)

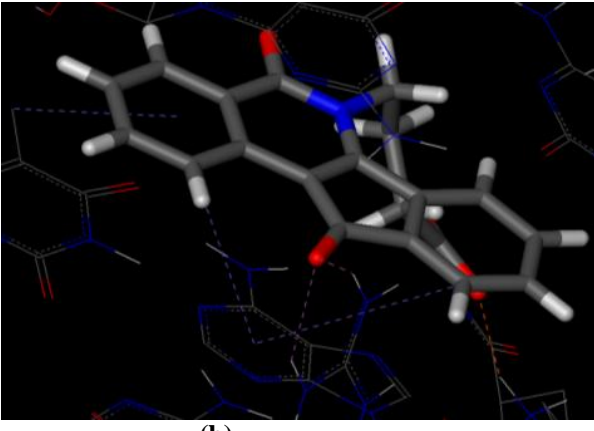

(b)

Fig.7a, b: 2D and 3D poses view of compound (VI) docked against topo I (PDB: 1SC7). In 2D, red, orange or violet dashed line represents hydrogen bonding while; the green solid line represents hydrophobic interactions. In 3D, red dashed line represents hydrogen bonding while; violet dashed line represents hydrophobic interactions. 
Compound (9b) formed a bi-dentate hydrogen bond between 4oxo and $\mathrm{NH}_{2}$ of Arg 364 and this hydrogen bond was reported to be essential for all human topo I inhibitors (Staker et al., 2005) and this hydrogen bond is similar to that formed by compounds(I) and (VI). Also, hydrophobic interactions between both tolyl and benzylidene with adenine were found similar to compound (I) and (MJ238, VI). Similarly, compound (9e) formed one hydrogen bond between 4-oxo and $\mathrm{NH}_{2}$ of Arg 364. Moreover, hydrophobic interactions between both $p$-bromophenyl and benzylidene with adenine were observed. The docking results were consistent with the in vitro cytotoxicity and suggested that compounds (9b) and (9e) may exhibit their cytotoxicity in a mechanism similar to that of compound (I) and (VI) through stabilization of topo I-DNA cleavage complex resulting in cancer cell death.

\section{CONCLUSION}

We designed and synthesized novel 5-arylidene-3(substituted phenyl)-2-( $p$-tolylamino)-3,5-dihydro-4H-imidazol-4ones 9a-f based on the structure of a thioxoimidazolidinone derivative (I) that has been reported to have cytotoxic activity with human topo I inhibitory activity. Cytotoxicity of the synthesized compounds has been evaluated against PC-3 and MCF-7 cell lines along with normal lung fibroblast cell line (WI-38). Moreover, molecular docking study has been conducted in comparison with compound (I) and the reference ligand (VI). Our study aimed to develop a new scaffold that can be used to develop novel potent and safe topo I inhibitors can be used as anticancer agents. our study revealed that two compounds of 4-imidazolones $\mathbf{9 b}$, e possessed a significant cytotoxic activity in nmol range against both cancer cell lines with high selectivity index. These results were consistent with the docking results that showed that the binding interactions of these compounds to topo I are similar to that of compounds (I) and (VI) with binding scores more than (I) and slightly lower than (VI). Collectively, 4-imidazolones 9b, e are considered as promising compounds with selective cytotoxic activity against both PC-3 and MCF-7 cell lines require further investigations to develop potent topo I inhibitors that can be used as anticancer agents.

\section{ACKNOWLEDGEMENT}

The authors are grateful to Dr. Wagdy M. Eldehna for help in reviewing the manuscript and Abdullah A. Elgazar RPh. Faculty of pharmacy - Cairo University for help in molecular docking study.

\section{Financial support and sponsorship: Nil.}

Conflict of interest: The authors declare no conflict of interest.

\section{REFERENCES}

Abdellatif KR, Fadaly WA. New 1, 2-diaryl-4-substitutedbenzylidene-5-4H-imidazolone derivatives: design, synthesis and biological evaluation as potential anti-inflammatory and analgesic agents. Bioorg. Chem., 2017; 72:123-129.

Alonso C, Fuertes M, González M, Rubiales G, Tesauro C, Knudsen BR, Palacios F. Synthesis and biological evaluation of indeno [1, 5] naphthyridines as topoisomerase I (TopI) inhibitors with antiproliferative activity. Eur. J. Med. Chem., 2016; 115:179-190.

Bassetto M, Ferla S, Pertusati F, Kandil S, Westwell AD, Brancale A, McGuigan C. Design and synthesis of novel bicalutamide and enzalutamide derivatives as antiproliferative agents for the treatment of prostate cancer. Eur. J. Med. Chem., 2016; 118:230-243.

Biosolveit Leadit. avaiable at: http://www.Biosolveit.De/flexx/. [accessed: 10/Oct/2015]

Beck DE, Lv W, Abdelmalak M, Plescia CB, Agama K, Marchand C, Pommier Y, Cushman M. Synthesis and biological evaluation of new fluorinated and chlorinated indenoisoquinoline topoisomerase I poisons. Bioorg. Med. Chem., 2016; 24:1469-1479.

Cheng-Kang Peng TZ, Xing-Jun Xu, Yi-Qun Chang, Wen Hou, Kuo Lu, Hui, Lin P-HS, Jing Lin, Wei-Min Chen. Novel 4-(4-substituted amidobenzyl)furan-2(5H)-one derivatives as topoisomerase I inhibitors. Eur. J. Med. Chem., 2017; 127:187-199.

Denizot F, Lang R. Rapid colorimetric assay for cell growth and survival: modifications to the tetrazolium dye procedure giving improved sensitivity and reliability. J. immunol. Methods, 1986; 89:271-277.

Dhingra AK, Chopra B, Dass R, Mittal SK. Synthesis, antimicrobial and anti-inflammatory activities of some novel 5-substituted imidazolone analogs. Chinese Chemical Letters, 2016; 27:707-710.

Gauthier MP, Michaux C, Rolin S, Vastersaegher C, de Leval X, Julémont F, Pochet L, Masereel B. Synthesis, molecular modelling and enzymatic evaluation of $( \pm)$ 3, 5-diphenyl-2-thioxoimidazolidin-4-ones as new potential cyclooxygenase inhibitors. Bioorg. Med. Chem., 2006; 14:918-927.

Ge R, Zhao Q, Xie Z, Lu L, Guo Q, Li Z, Zhao L. Synthesis and biological evaluation of 6-fluoro-3-phenyl-7-piperazinyl quinolone derivatives as potential topoisomerase I inhibitors. Eur. J. Med. Chem., 2016; 122:465-474.

Hou W, Wang Z-Y, Peng C-K, Lin J, Liu X, Chang Y-Q, Xu J, Jiang $\mathrm{R}-\mathrm{W}$, Lin $\mathrm{H}$, Sun $\mathrm{P}-\mathrm{H}$. Novel securinine derivatives as topoisomerase I based antitumor agents. Eur. J. Med. Chem., 2016; 122:149-163.

Jeon K-H, Yu H-B, Kwak SY, Kwon Y, Na Y. Synthesis and topoisomerases inhibitory activity of heteroaromatic chalcones. Bioorg. Med. Chem., 2016; 24:5921-5928.

Jung M-H, El-Gamal MI, Abdel-Maksoud MS, Sim T, Yoo KH, Oh C-H. Design, synthesis, and antiproliferative activity of new $1 \mathrm{H}-$ pyrrolo [3, 2-c] pyridine derivatives against melanoma cell lines. Part 2. Bioorg. Med. Chem. Lett., 2012; 22:4362-4367.

Jung ME, Ouk S, Yoo D, Sawyers CL, Chen C, Tran C, Wongvipat J. Structure- activity relationship for thiohydantoin androgen receptor antagonists for castration-resistant prostate cancer (CRPC). J. Med. Chem., 2010; 53:2779-2796.

Kamal A, Ramakrishna G, Raju P, Viswanath A, Ramaiah MJ, Balakishan G, Pal-Bhadra M. Synthesis and anti-cancer activity of chalcone linked imidazolones. Bioorg. Med. Chem. Lett., 2010; 20:48654869 .

Khatik GL, Kaur J, Kumar V, Tikoo K, Venugopalan P, Nair VA. Aldol derivatives of Thioxoimidazolidinones as potential anti-prostate cancer agents. Eur. J. Med. Chem., 2011; 46:3291-3301.

Khodair AI. Glycosylation of 2-thiohydantoin derivatives. Synthesis of some novel S-alkylated and S-glucosylated hydantoins. Carbohydr. Res., 2001; 331:445-453.

Khodair AI, El-Subbagh H, Al-Obaid A. Synthesis, conformational analysis and antitumor testing of 5-(Z)-arylidene-4imidazolidinone derivatives. Phosphorus, Sulfur Silicon Relat. Elem., 1998; 140:159-181.

Kumar D, Mariappan G, Husain A, Monga J, Kumar S. Design, synthesis and cytotoxic evaluation of novel imidazolone fused quinazolinone derivatives. Arabian Journal of Chemistry, 2014.

Majumdar P, Bathula C, Basu SM, Das SK, Agarwal R, Hati S, Singh A, Sen S, Das BB. Design, synthesis and evaluation of 
thiohydantoin derivatives as potent topoisomerase I (Top1) inhibitors with anticancer activity. Eur. J. Med. Chem., 2015; 102:540-551.

Mauceri HJ, Hanna NN, Beckett MA, Gorski DH, Staba M-J, Stellato KA, Bigelow K, Heimann R, Gately S, Dhanabal M. Combined effects of angiostatin and ionizing radiation in antitumour therapy. Nature, 1998; 394:287-291.

Protein data bank. avaiable at: http://www.rcsb.org. [accessed: 10/Oct/2015].

Mazza A, Beccalli EM, Contini A, Garcia-Argaez AN, Dalla Via L, Gelmi ML. A new scaffold of topoisomerase I inhibitors: Design, synthesis and biological evaluation. Eur. J. Med. Chem., 2016; 124:326339.

MOE. Chemical Computing Group 1, Montreal,. MOE. Chemical Computing Group, lnc., Montreal,

Park S, Magar TBT, Kadayat TM, Lee HJ, Bist G, Shrestha A, Lee E-S, Kwon Y. Rational design, synthesis, and evaluation of novel 2, 4-Chloro-and Hydroxy-Substituted diphenyl Benzofuro [3, 2-b] Pyridines: Non-intercalative catalytic topoisomerase I and II dual inhibitor. Eur. J. Med. Chem., 2017; 127:318-333.

Siegel RL, Miller KD, Jemal A. Cancer statistics, 2016. C.Cancer j. Clin., 2016; 66:7-30.

Spicer JA, Lena G, Lyons DM, Huttunen KM, Miller CK, O’Connor PD, Bull M, Helsby N, Jamieson SM, Denny WA. Exploration of a series of 5-arylidene-2-thioxoimidazolidin-4-ones as inhibitors of the cytolytic protein perforin. J. Med. Chem., 2013; 56:9542-9555.

Staker BL, Feese MD, Cushman M, Pommier Y, Zembower D, Stewart L, Burgin AB. Structures of three classes of anticancer agents bound to the human topoisomerase I-DNA covalent complex. J. Med. Chem., 2005; 48:2336-2345.

Stierand K, Rarey M. From Modeling to Medicinal Chemistry: Automatic Generation of Two-Dimensional Complex Diagrams. Chem Med Chem., 2007; 2:853-860.

Stierand K, Rarey M. PoseView--molecular interaction patterns at a glance. J. Cheminformatics, 2010; 2:1-1.
Das SK, Rehman I, Ghosh A, Sengupta S, Majumdar P,Jana B, Das BB. Poly(ADP-ribose) polymers regulate DNA topoisomerase I (Top1) nuclear dynamics and camptothecin sensitivity in living cells. Nucleic Acids Res., 2016; 44:8363-8375.

Subtel'na I, Atamanyuk D, Szymańska E, Kieć-Kononowicz K, Zimenkovsky B, Vasylenko O, Gzella A, Lesyk R. Synthesis of 5arylidene-2-amino-4-azolones and evaluation of their anticancer activity. Bioorg. Med. Chem., 2010; 18:5090-5102.

Topcu Z. DNA topoisomerases as targets for anticancer drugs. J. Clin. Pharm. Ther., 2001; 26:405-416.

Available

World Cancer Report 2014, World Health Organization.

http://apps. who.int/bookorders/anglais/detart1.jsp?codlan=1\&codcol=76\& codcch $=31$, [accessed 23/Dec/2016].

Zhang C, Qu Y, Niu B. Design, synthesis and biological evaluation of lapachol derivatives possessing indole scaffolds as topoisomerase I inhibitors. Bioorg. Med. Chem., 2016; 24:5781-5786.

\section{How to cite this article:}

Khodair AI, Elbadawi MM, Elsaady MT, Abdellatif KRA. Design, synthesis, molecular docking and cytotoxicity evaluation of some novel 5-arylidene-3-(substituted phenyl)-2-( $p$-tolylamino)-4imidazolones. J App Pharm Sci, 2017; 7 (09): 058-068. 